\title{
DIFFERENT SYNCHRONIZATION REGIMES IN A SYSTEM OF THREE COUPLED 1-D MAPS
}

\author{
M.Hasler*, O.Popovych** and Y.Maistrenko** \\ *Department of Electrical Engineering \\ Swiss Federal Institute of Technology \\ 1015 Lausanne, Switzerland \\ fax: +41 21693 6700, e-mail: martin.hasler@epfl.ch \\ ** Institute of Mathematics, \\ NationalAcademy of Sciences of Ukraine \\ Kiev, Ukraine \\ fax: +380 4422520 10, e-mail: maistrenko@imath.kiev.ua
}

Keywords: Chaos, synchronization, coupled maps, attractor,

\section{ABSTRACT}

The notions of strong and weak synchronization of chaotic systems are reviewed in the context of three coupled identical one-dimensional maps. Two possibilities exist. Either all three systems synchronize (total synchronization), or only two out of three systems synchronize (partial synchronization, or clustering). In either case, strong and weak synchronization is possible.

\section{INTRODUCTION}

Synchronization of two identical chaotic dynamical systems has received much attention in the mathematics and physics literature in the last few years [1-8]. The most advanced application in engineering is the transmission of information on a chaotic carrier signal, where the receiver is synchronized with the transmitter [9]. One can distinguish essentially two synchronization regimes, strong synchronization and weak synchronization [10]. We will first review these concepts. When three identical dynamical systems are coupled, a new phenomenon may be observed, namely partial chaotic synchronization, also called clustering, where only two of the three dynamical systems synchronize. Partial synchronization may also be weak or strong, as we shall point out. The coupling of the three systems may be symmetric or not, which has some influence on the synchronization behavior. Different cases will be discussed and illustrated.

\section{THREE COUPLED MAPS}

We consider the iteration of three coupled the skew tent maps:

$$
\mathbf{x}_{\mathrm{n}+1}=\mathbf{F}\left(\mathbf{x}_{\mathrm{n}}\right), \quad \mathbf{F}: \mathfrak{R}^{3} \rightarrow \mathfrak{R}^{3}
$$

where

$$
\mathbf{F}(\mathbf{x})=\mathbf{f}(\mathbf{A x}), \quad \mathbf{f}\left(\begin{array}{l}
x \\
y \\
z
\end{array}\right)=\left(\begin{array}{c}
f(x) \\
f(y) \\
f(z)
\end{array}\right)
$$

A is a matrix, the coupling matrix, and the function $\mathrm{f}$ is the skew tent map

$$
f(x)=\left\{\begin{array}{cc}
b \frac{x}{a} & \text { for } x \leq a \\
b \frac{1-x}{1-a} & \text { for } x>a
\end{array}\right.
$$

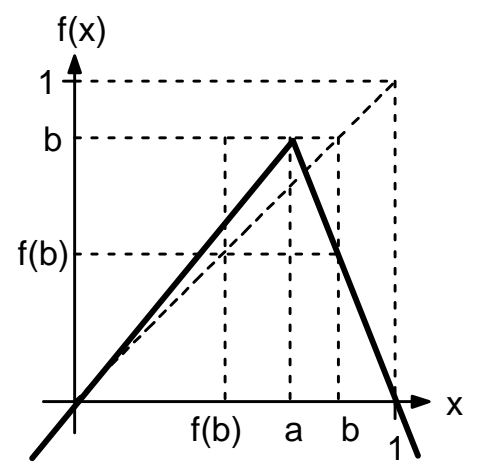

Fig.1. Skew tent map

This function is determined by the two parameters $a$ and $b$ which we restrict to

$$
0<\mathrm{a}<1, \quad \max \{\mathrm{a}, 1-\mathrm{a}\}<\mathrm{b} \leq 1
$$

The one-dimensional dynamical system generated by the iterations of $\mathrm{f}$ has the attracting invariant interval $\mathrm{I}=$ $[f(b), b]$ whose basin of attraction is the open interval $(0,1)$, and on $I$ it has chaotic behavior, because the slopes of $f$ are always larger than 1. Furthermore, it has a natural invariant measure $\mu$ on I with density $\rho(x)[11]$. 
We shall consider in particular two examples for the coupling matrix A:

\section{Example 1: Symmetric coupling}

$$
\mathbf{A}=\left(\begin{array}{ccc}
1-2 \varepsilon & \varepsilon & \varepsilon \\
\varepsilon & 1-2 \varepsilon & \varepsilon \\
\varepsilon & \varepsilon & 1-2 \varepsilon
\end{array}\right)
$$

Example 2: Nonsymmetric coupling

$$
\mathbf{A}=\left(\begin{array}{ccc}
1-\varepsilon & 0 & \varepsilon \\
0 & 1-\varepsilon & \varepsilon \\
\varepsilon & 0 & 1-\varepsilon
\end{array}\right)
$$

\section{STRONG TOTAL SYNCHRONIZATION}

The matrix of coupling coefficients $\mathbf{A}$ is supposed to satisfy

$$
\sum_{j=1}^{3} A_{i j}=1
$$

which is the case for the two examples. This implies that the main diagonal

$$
\mathrm{D}=\{(\mathrm{x}, \mathrm{y}, \mathrm{z}) \mid \mathrm{x}=\mathrm{y}=\mathrm{z}\}
$$

is invariant under the three-dimensional dynamics (1). Note that the three-dimensional dynamics (1) restricted to $\mathrm{D}$ is equivalent to the one-dimensional dynamics given by the iterations of $f$ on the real line.

We say that the three coupled systems synchronize, or that the system (1) synchronizes totally, if the trajectory remains bounded and converges to the main diagonal D, i.e. if

$$
\left|\mathrm{x}_{\mathrm{n}}-\mathrm{y}_{\mathrm{n}}\right|_{\mathrm{n} \rightarrow \infty}^{\rightarrow} 0 \text { and }\left|\mathrm{x}_{\mathrm{n}}-\mathrm{z}_{\mathrm{n}}\right|_{\mathrm{n} \rightarrow \infty}^{\rightarrow} 0
$$

This property depends in general on the trajectory, i.e. on the initial conditions.

\section{Definition 1:}

Let $\mathrm{V}$ be a bounded open set in $\mathfrak{R}^{3}$ that is invariant under the dynamics (1) and whose intersection with the main diagonal D is non empty. The system has strong total synchronization in $V$ if the following conditions are satisfied:

- all trajectories starting in V synchronize totally.

- $\quad$ the intersection of $\mathrm{V}$ and $\mathrm{D}$ is Lyapunov stable.

\section{Remark:}

Under strong synchronization, if $\mathrm{A}$ is an attractor for the 1$\mathrm{d}$ dynamics in the intersection of $\mathrm{V}$ and $\mathrm{D}$, then $\mathrm{A}$ is also attractor for the 3-d dynamics (in the usual sense).

\section{Example 1:}

Let the coupling matrix be given by (5). Then by a Lyapunov similar to [10] we can prove that all trajectories converge towards the diagonal if

$$
\mathrm{b} \max \left(\frac{1}{\mathrm{a}}, \frac{1}{1-\mathrm{a}}\right) \cdot|1-3 \varepsilon|<1
$$

In order to establish strong synchronization, we still have to exhibit an open bounded invariant set that contains part of the diagonal D. Otherwise, the trajectories may go to infinity along the diagonal $\mathrm{D}$.

It is not difficult to show that for sufficiently small $\eta$ the open set defined by the six inequalities

$$
\begin{array}{ll}
\mathrm{f}(\mathrm{b})-\eta<\mathrm{x}<\mathrm{b}+\eta, & |\mathrm{x}-\mathrm{y}|<\eta \\
\mathrm{f}(\mathrm{b})-\eta<\mathrm{y}<\mathrm{b}+\eta, & |\mathrm{x}-\mathrm{z}|<\eta \\
\mathrm{f}(\mathrm{b})-\eta<\mathrm{z}<\mathrm{b}+\eta, & |\mathrm{y}-\mathrm{z}|<\eta
\end{array}
$$

is invariant under $\mathbf{F}$.

Note that the synchronization error

$$
\mathrm{e}_{\mathrm{n}}=\max \left\{\mathrm{x}_{\mathrm{n}}-\mathrm{y}_{\mathrm{n}}|,| \mathrm{x}_{\mathrm{n}}-\mathrm{z}_{\mathrm{n}}|,| \mathrm{y}_{\mathrm{n}}-\mathrm{z}_{\mathrm{n}} \mid\right\}
$$

is actually decreasing exponentially fast. A typical trajectory with the corresponding synchronization error is represented in Fig.2.
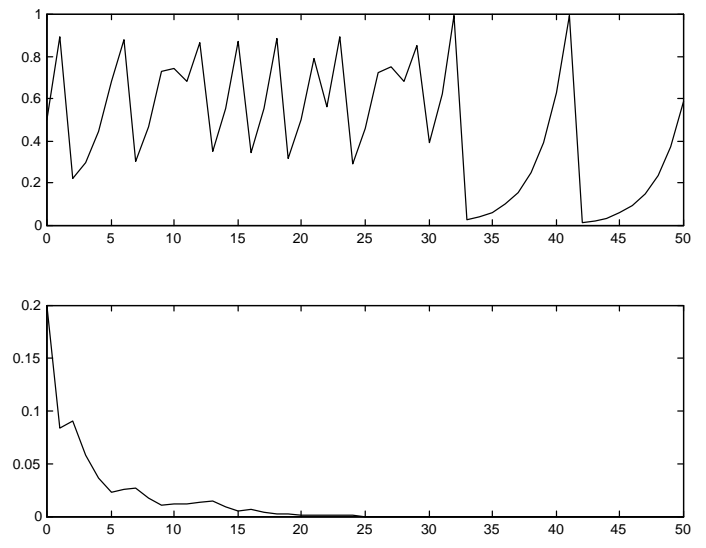

Fig.2. Strong total synchronization in the symmetrically coupled maps. Upper part: 50 samples of the x-trajectory, lower part: 50 samples of the synchronization error. Parameters: $\mathrm{a}=0.63, \mathrm{~b}=0.68, \varepsilon=0.2$. Initial conditions: $\mathrm{x}_{0}=0.5, \mathrm{y}_{0}=0.6, \mathrm{z}_{0}=0.7$.

\section{Example 2:}

Let the coupling matrix be given by (6). Similarly to example1, one obtains strong synchronization for 


$$
1-\frac{1}{\mathrm{~K}}<\varepsilon<\frac{1}{2}+\frac{1}{2 \mathrm{~K}}
$$

with

$$
\mathrm{K}=\mathrm{b} \max \left(\frac{1}{\mathrm{a}}, \frac{1}{1-\mathrm{a}}\right)
$$

\section{Remark:}

Instead of the global Lyapunov function approach we may check whether a local method leads to a larger region of strong synchronization in parameter space.

A necessary local condition for strong synchronization in some neighborhood of $\mathrm{I}$ is that the transversal Lyapunov exponents of all synchronized trajectories are nonpositive.

\section{Example 1:}

The Jacobian of $\mathbf{F}$ in the linear region $\mathrm{x}=\mathrm{y}=\mathrm{z}<\mathrm{a}$ has the eigenvalue $\mathrm{b} / \mathrm{a}$ with the eigenvector $(1,1,1)$ and $(1-3 \varepsilon) \mathrm{b} / \mathrm{a}$ with all vectors orthogonal to $(1,1,1)$ as eigenvectors. In the linear region $\mathrm{x}=\mathrm{y}=\mathrm{z}>\mathrm{a}$ the eigenvalues are, respectively, $\quad \mathrm{b} /(1-\mathrm{a})$ and $(1-3 \varepsilon) \mathrm{b} /(1-\mathrm{a}), \quad$ and the eigenvectors are the same as in the other linear region.

In the case of a $>0.5$, the transversal eigenvalue (1$3 \varepsilon) b /(1-a)$ is larger than the transversal eigenvalue (1$3 \varepsilon) b / a$. Furthermore, the fixed point of $\mathrm{f}$ in $\mathrm{I}$ has (1$3 \varepsilon) b /(1-a)$ as an eigenvalue and thus the trajectory with the largest transversal Lyapunov exponent is the constant trajectory that stays at that fixed point. Hence, from this local analysis for a $>0.5$, we get essentially the same condition (10) as from the global analysis.

In the case of a $<0.5$, the trajectory in D with the largest transversal Lyapunov exponent is periodic of maximal type with a period that depends on $b$. It passes in both linear regions and therefore its transversal Lyapunov exponent is smaller than $\ln ((1-3 \varepsilon) \mathrm{b} / \mathrm{a})$. In this case we get an indication of a slightly larger region of strong synchronization from the local analysis than from the global analysis.

\section{Example 2:}

Analogous to example 1.

\section{WEAK TOTAL SYNCHRONIZATION}

As opposed to strong synchronization, weak synchronization does not require synchronization of all trajectories in a neighborhood of the diagonal.

\section{Definition 2:}

The system has weak total synchronization if there is a bounded closed indecomposable non-empty set $\mathrm{B}$ on the diagonal $\mathrm{D}$ which satisfies the following conditions:
- $\quad$ B is invariant under the one-dimensional dynamics on D obtained by restricting (1) to D.

- The set of initial conditions in $\Re^{3}$ that give rise to trajectories converging to $\mathrm{B}$ has positive Lebesgue measure.

- B is not Lyapunov asymptotically stable under the three-dimensional dynamics (1).

\section{Remark:}

Under weak synchronization, attractor B on D is an attractor in Milnor sense according to dynamical systems theory terminology.

\section{Theorem:[1,5]}

Let $\mathrm{B}$ be as in definition 2. If both natural transversal Lyapunov exponents of the trajectories in B are negative, the system has weak total synchronization.

\section{Example 1:}

Given the transversal eigenvalues $(1-3 \varepsilon$ b/a and $(1-3 \varepsilon) b /(1-$ a) of the linear regions on the diagonal, it follows that the natural transversal Lyapunov exponents are

$\lambda_{\perp}^{(\mathrm{nat})}=\ln |1-3 \varepsilon|+\mathrm{m} * \ln \left(\frac{\mathrm{b}}{\mathrm{a}}\right)+(1-\mathrm{m} *) \ln \left(\frac{\mathrm{b}}{1-\mathrm{a}}\right)$

where $\mathrm{m}^{*}$ is the natural invariant measure of the interval [f(b),a] under the iterations of $f$. Only for special parameter values this measure can be determined explicitly, for the others it has to be determined numerically Fig. 3 .

If the natural transversal Lyapunov exponent is negative, but condition (10) for strong synchronization is not satisfied, then typical synchronizing trajectories do not approach the diagonal uniformly, but through a sequence of bursts of varying scales of the synchronization errors (Fig.4). Depending on the global behavior of the nonlinear dynamics, the nonsynchronizing trajectories may simply show an infinite sequence of desynchronization bursts, or they may converge to some attractor in $\mathrm{V}$ off the diagonal, or they may diverge to -infinity.

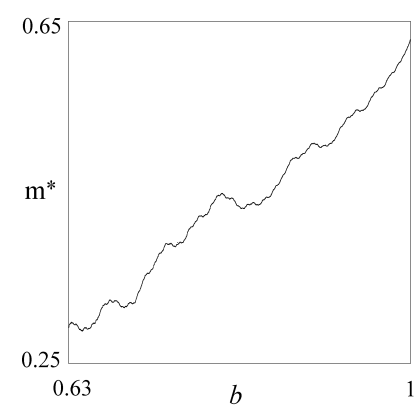

Fig.3. Natural invariant measure of the interval $[\mathrm{f}(\mathrm{b}), \mathrm{a}]$ as a function of $b$. Parameter $a=0.63$. 

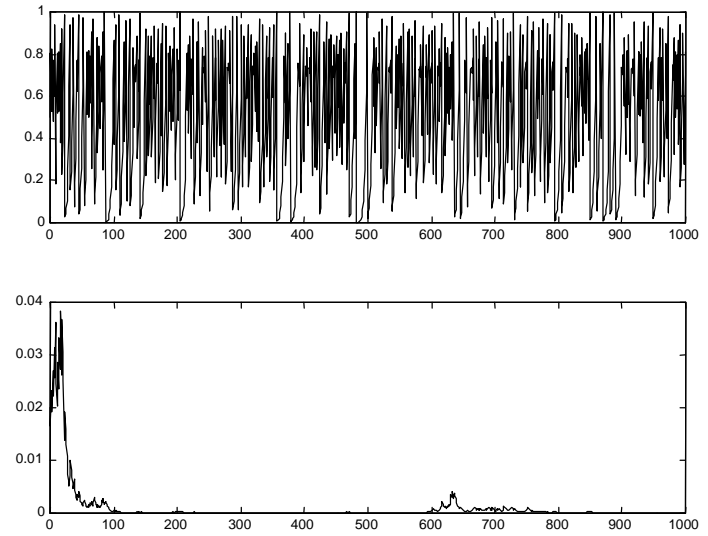

Fig.4. Weak total synchronization in the symmetrically coupled maps. Upper part: 1000 samples of the $\mathrm{X}$ trajectory, lower part: 1000 samples of the synchronization error. Parameters: $\mathrm{a}=0.63, \mathrm{~b}=0.68, \varepsilon=0.16$. Initial conditions: $\mathrm{x}_{0}=0.5, \mathrm{y}_{0}=0.51, \mathrm{z}_{0}=0.52$.

\section{Remark:}

If the coupled systems are not perfectly identical, or if the system is perturbed by small noise, the phenomenon of strong and weak synchronization become very different [10]. While strongly synchronizing systems still approximately synchronize under these perturbations, weakly synchronizing systems usually show a never ending sequence of large desynchronization bursts, or they desynchronize completely, due to some other attractor off the diagonal.

\section{Example 2:}

The behavior is similar to example 1 .

\section{STRONG PARTIAL SYNCHRONIZATION}

It is possible that of the three coupled systems only two synchronize. We call this phenomenon partial synchronization. In the papers where a large number of coupled systems are discussed, the same phenomenon is called clustering.

A prerequisite for partial synchronization of the systems with state variables, say, $\mathrm{x}$ and $\mathrm{y}$ is that the plane

$$
\Pi_{x y}=\{(x, y, z) \mid x=y\}
$$

is invariant under the dynamics (1). It is easy to see that this is equivalent to the constraint $A_{13}=A_{23}$. Similarly for the planes $\Pi_{\mathrm{xz}}$ and $\Pi_{\mathrm{yz}}$. In example 1 , all three planes are invariant, whereas in example 2 , the planes $\Pi_{x y}$ and $\Pi_{x z}$ are invariant, while the plane $\Pi_{\mathrm{yz}}$ is not.
The main condition for $\mathrm{x}-\mathrm{y}$ partial synchronization is that

$$
\left|x_{n}-y_{n}\right| \underset{n \rightarrow \infty}{\rightarrow} 0
$$

but the difference between $x_{n}$ and $z_{n}$ does not converge to zero. Similarly for $\mathrm{x}-\mathrm{z}$ and $\mathrm{y}-\mathrm{z}$ partial synchronization.

\section{Definition 3:}

Suppose that the plane $\Pi_{x y}$ is invariant under the dynamics (1). Let $\mathrm{V}$ be an invariant bounded open set in $\mathfrak{R}^{3}$ whose intersection with $\Pi_{\mathrm{xy}}$ is non empty. The system has strong partial synchronization of the variables $x$ and $y$ in $V$ if the following conditions are satisfied:

- $\quad$ all trajectories starting in $V$ converge to $\Pi_{x y}$.

- $\quad$ the intersection of $V$ and $\Pi_{x y}$ is Lyapunov stable.

- the system has no strong total synchronization in $\mathrm{V}$ nor has it weak total synchronization.

Similarly for strong partial synchronization of the other pairs of variables.

\section{Example 2:}

Recall that the trajectories converge to the plane $\Pi_{x y}$ if

$$
\mathrm{b} \max \left(\frac{1}{\mathrm{a}}, \frac{1}{1-\mathrm{a}}\right)|1-\varepsilon|<1
$$

and to the plane $\Pi_{\mathrm{xz}}$ if

$$
\mathrm{b} \max \left(\frac{1}{\mathrm{a}}, \frac{1}{1-\mathrm{a}}\right)|1-2 \varepsilon|<1
$$

If both conditions are satisfied, we have strong total synchronization. Thus, for strong partial $\mathrm{x}-\mathrm{y}$ synchronization, (18) has to be satisfied and (19) has to be violated.

Consider the dynamical system restricted to the plane $\Pi_{x y}$ :

$$
\begin{aligned}
& \mathrm{x}_{\mathrm{n}+1}=\mathrm{f}\left(\mathrm{x}_{\mathrm{n}}+\varepsilon\left(\mathrm{z}_{\mathrm{n}}-\mathrm{x}_{\mathrm{n}}\right)\right) \\
& \mathrm{z}_{\mathrm{n}+1}=\mathrm{f}\left(\mathrm{z}_{\mathrm{n}}+\varepsilon\left(\mathrm{x}_{\mathrm{n}}-\mathrm{z}_{\mathrm{n}}\right)\right)
\end{aligned}
$$

In order to avoid also weak total synchronization, the natural transversal Lyapunov exponent to the diagonal $\mathrm{x}=$ $\mathrm{z}$ in the plane $\Pi_{\mathrm{xy}}$ must be positive. After some calculation one obtains

$\lambda_{\perp x y}^{(\text {nat })}=\ln |1-2 \varepsilon|+m * \ln \left(\frac{b}{a}\right)+\left(1-m^{*}\right) \ln \left(\frac{b}{1-a}\right)$

Since the positivity of (21) is more restrictive than the violation of (19), the conditions for strong partial $x-y$ 
synchronization are (18) and (21). An example is given in Figs.5,6.
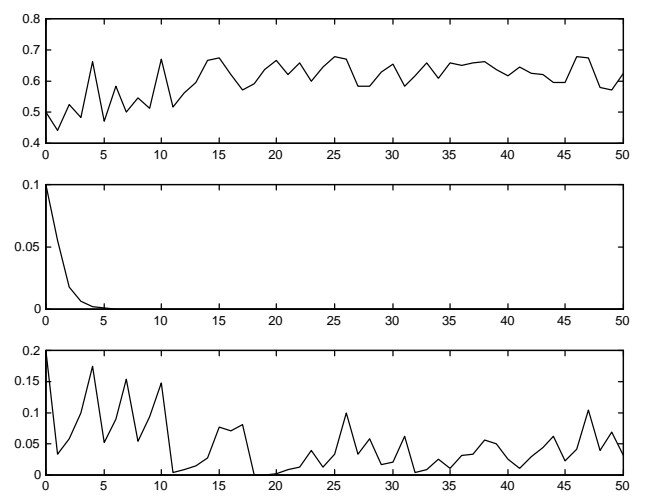

Fig.5. Strong partial $x-y$ synchronization in the non symmetrically coupled maps. Upper part: 50 samples of the $\mathrm{x}$-trajectory, middle part: 50 samples of the $\mathrm{x}-\mathrm{y}$ synchronization error, lower part: : 50 samples of the $\mathrm{x}-\mathrm{z}$ synchronization error. Parameters: $\mathrm{a}=0.63, \mathrm{~b}=0.68, \varepsilon=$ 1.3. Initial conditions: $\mathrm{x}_{0}=0.5, \mathrm{y}_{0}=0.6, \mathrm{z}_{0}=0.7$.

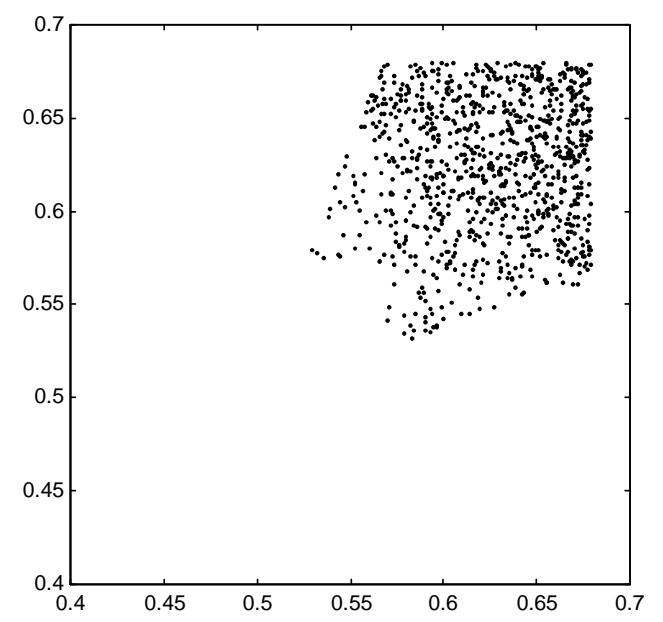

Fig.6. 1000 point of trajectory of Fig.5 in the $\mathrm{x}=\mathrm{y}$ plane. The first 50 points are not represented.

\section{Example 1:}

It has been proved that as soon as all three planes are invariant, asymptotic stability of one of the planes implies asymptotic stability of the main diagonal [13]. Therefore, strong partial synchronization in an invariant region $\mathrm{V}$ that contains part of the main diagonal is not possible. However, if the intersection of $\mathrm{V}$ with the plane $\Pi_{\mathrm{xy}}$ does not intersect the diagonal, strong partial $x-y$ synchronization is even possible in the symmetrically coupled system. Note that by symmetry, there are simultaneously also attractors in the $x-z$ and in the $y-z$ plane. Depending on the initial conditions, a trajectory $x-y$, $\mathrm{x}-\mathrm{z}$, or $\mathrm{y}-\mathrm{z}$ synchronizes. The basins of attraction of the three attractors have a very complicated geometric structure. However, there is a small open set around each attractor that belongs entirely to the basin, as required by asymptotic stability.
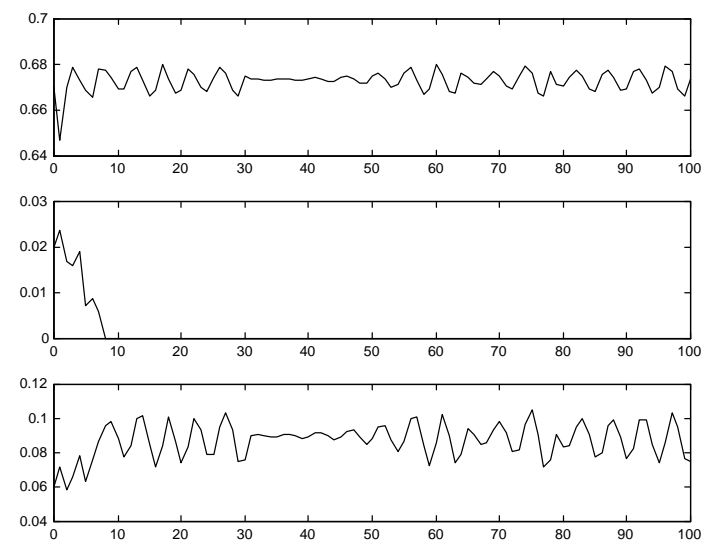

Fig.7. Strong partial $x-y$ synchronization in the symmetrically coupled maps. Upper part: 100 samples of the $\mathrm{x}$-trajectory, middle part: 100 samples of the $\mathrm{x}-\mathrm{y}$ synchronization error, lower part: : 100 samples of the $\mathrm{x}-\mathrm{Z}$ synchronization error. Parameters: $\mathrm{a}=0.63, \mathrm{~b}=0.68, \varepsilon=$ 0.55. Initial conditions: $\mathrm{x}_{0}=0.67, \mathrm{y}_{0}=0.69, \mathrm{z}_{0}=0.61$.

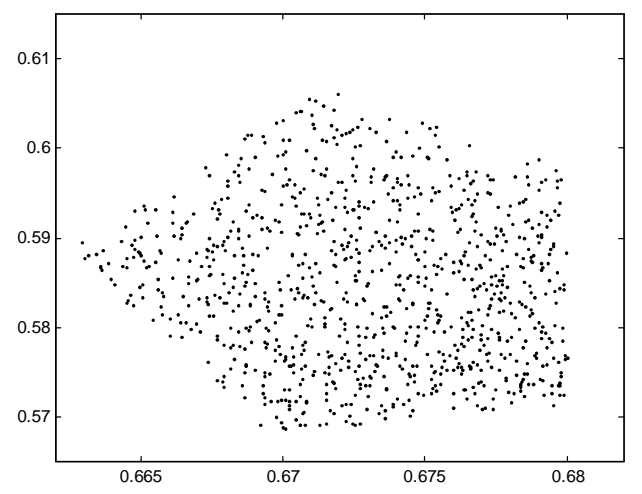

Fig. 8. 1000 points of the trajectory of Fig.7 in the $x=y$ plane. Note that the attractor does not intersect the diagonal. The first 50 points of the trajectory are not represented

\section{WEAK PARTIAL SYNCHRONIZATION}

\section{Definition 4:}

The system has weak partial synchronization of the variables $x$ and $y$ if there is a bounded closed indecomposable non-empty set $B$ in the plane $\Pi_{x y}$ which satisfies the following conditions:

- $\quad$ B is invariant under the two-dimensional dynamics on $\Pi_{x y}$ obtained by restricting (1) to $\Pi_{x y}$.

- The set of initial conditions in $\mathfrak{R}^{3}$ that give rise to trajectories converging to $\mathrm{B}$ has positive Lebesgue measure. 
- B is not Lyapunov asymptotically stable under the three-dimensional dynamics (1).

Similarly for weak partial synchronization of the other pairs of variables. Again, the criterion for weak partial $x-y$ synchronization is a negative $\Pi_{x y}$-transversal Lyapunov exponent for trajectories in B.

\section{Example 2:}

In example 2, there is no weak $\mathrm{x}-\mathrm{y}$ synchronization, because the trajectories diverge to infinity before condition (18) is violated. However, weak $\mathrm{X}-\mathrm{z}$ synchronization is possible (Figs.9,10).
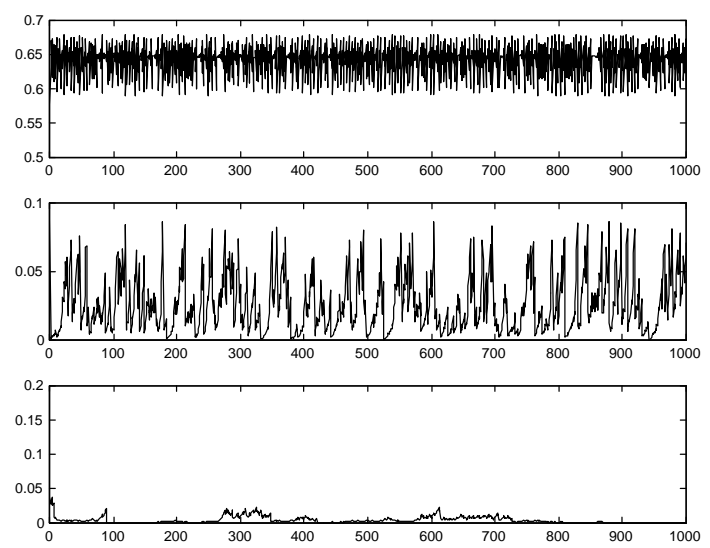

Fig.9. Weak partial $\mathrm{x}-\mathrm{z}$ synchronization in the non symmetrically coupled maps. Upper part: 1000 samples of the $\mathrm{x}$-trajectory, middle part: 1000 samples of the $x-y$ synchronization error, lower part: 1000 samples of the $\mathrm{X}-\mathrm{Z}$ synchronization error. Parameters: $\mathrm{a}=0.63, \mathrm{~b}=$ $0.68, \varepsilon=0.175$. Initial conditions: $\mathrm{x}_{0}=0.5, \mathrm{y}_{0}=$ $0.6, \mathrm{z}_{0}=0.7$.

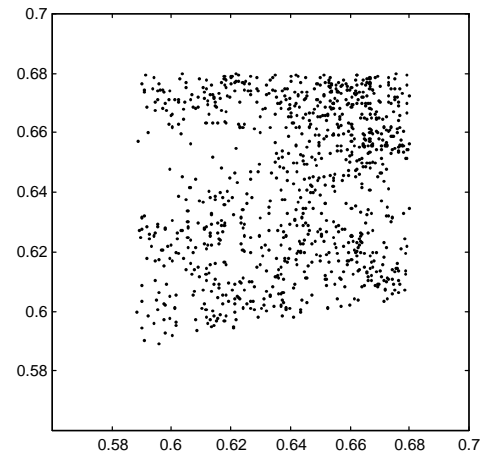

Fig.10. 1000 points of the trajectory of Fig.9 in the $\mathrm{x}=\mathrm{z}$ plane. The first 50 points are not represented.

\section{CONCLUSION}

The dynamical system generated by the iterations of three coupled one-dimensional maps has been considered. The notions of strong and weak total and partial synchronization have been defined. Two different coupling matrices have been used to illustrate the different kinds of synchronization, one symmetric and the other non symmetric. All cases are possible for both examples, except that for symmetric coupling where strong partial synchronization can only exist with attractors away from the main diagonal. Most cases have been illustrated by concrete examples.

\section{REFERENCES}

[1] J.C.Alexander, J.A.Yorke, Z.You, "Riddled basins", Int. J. of Bif. and Chaos, vol. 2, pp.795-813, 1992.

[2] P.Ashwin, J.Buescu, I.Stewart, "Bubbling of attractors and synchronization of chaotic oscillators", Phys. Lett. A, vol. 193, pp.126-139, 1994.

[3] E.Ott, J.C.Sommerer, "Blowout bifurcations: the occurrence of riddled basins", Phys. Lett.A, vol. 188, pp.39-47, 1994.

[4] Y-C.Lai, C.Grebogi, J.A.Yorke, "Riddling bifurcation in chaotic dynamical systems", Phys. Rev. Lett, vol. 77, 1996, pp.55-58, 1996.

[5] P.Ashwin, J.Buescu, I.Stewart, "From attractor to chaotic saddle: a tale of transverse instability", Nonlinearity, vol.9, pp.703-737, 1996.

[6] Y.Maistrenko, T.Kapitaniak, "Different types of chaos synchronization in two coupled piecewise linear maps", Phys. Rev.E., vol.54, pp.3285-3292, 1996.

[7] J.F.Haegy, T.L.Carroll, L.Peccora, "Experimental and numerical evidence for riddled basins in coupled chaotic systems", Phys. Rev. Lett., vol. 73, pp.35283531, 1994.

[8] L.M.Pecora, T.L.Carroll, "Synchronization in Chaotic Systems", Phys. Rev. Lett., vol.64, pp.821-824, 1990.

[9] M.Hasler, "Engineering chaos for encryption and broadband communication", to appear in Philosophical Transactions of the Royal Society of London, Trransaction A, vol.353, pp.115-126, 1995.

[10] M.Hasler, Y.Maistrenko, "An introduction to the synchronization of chaotic systems: coupled skew tent maps", IEEE Trans.Circ.Syst.-Part I, vol.44, pp.856866., Oct.1997.

[11] W.De Melo, S. Van Strien, One-dimensional dynamics, Springer Verlag, Berlin, 1993.

[12] K.Kaneko, "Clustering, coding, switching hierarchical ordering, and control in a network of chaotic elements", Physica D41, pp.137-172, 1990.

[13] Y.Maistrenko, O.Popovich, M.Hasler, "On strong and weak chaotic partial synchronization", to be published in the Intern. J. on Bif. and Chaos. 